\title{
The Design and Implementation of SIP Multi-domain SmartHome System
}

\author{
Lianfen Huang ${ }^{1, a}$, Jianyuan Liu ${ }^{1, b}$, Zhichao Huang $^{1, c}$, Bingbing Ding ${ }^{1, d}$, Hezhi \\ $\operatorname{Lin}^{1,}$ e \\ ${ }^{1}$ College of Information Science and Technology, Xiamen University, Xiamen, China \\ alfhuang@xmu.edu.cn, ${ }^{b} l j y 0811 @ g m a i l . c o m, ~{ }^{c} 412707666 @ q q . c o m,{ }^{d} 524554217 @ q q . c o m,{ }^{e}$ linhez \\ hi@xmu.edu.cn
}

Keywords: SmartHome, SIP, multi-domain, SIP server, redirect

\begin{abstract}
Heretofore, most of SmartHome systems deliver related services basing on a family local area network, and are not yet mature in remote control. In connection with this problem, we propose a SIP multi-domain system with distributed architecture, extend SIP protocol's redirect registration and call, make a combination of SmartHome system and the internet, and help people access to their home internal networking whenever and wherever.
\end{abstract}

\section{Introduction}

With the explosive growth of embedded electronics and automation equipment, Internet of Things (IoT) based on ad-hoc network is developing rapidly, and then provides people with all kinds of efficient and convenient application. As family ad-hoc network technology becomes more mature, such as zigbee, wifi, bluetooth, Ethernet and power line cable network technology, smart homes, being an important application field of IoT, also offers a variety of applications, such as visual intercom, home appliance control, intelligent lighting, etc. However, the intelligent network era of smart home is coming soon because of the rapid development of network technology in recent years, which developing to IP-centered multimedia business integrated network and presenting ubiquitous, fusion, open, integration [1]. This paper, based on the new features of smart home, using the SIP protocol, building SIP server and multiple domain management system, realize the home network and the Internet.

\section{SIP Protocol and its mobility}

SIP (Session Initiation Protocol) is a VOIP protocol made by IETF (Internet Engineering Task Force) to establish, modify and terminate multimedia sessions. It's an important protocol of soft switching system and IMS (IP Multimedia Subsystem) in NGN (Next Generation Network) [2]. Similar to the HTTP protocol, SIP use the UTF-8 character set to encode, which is not only easier to read and understand, but also easy to extend [3]. In applications, it usually provides one basis to implement diffident function. A complex SIP system could have more than one SIP management area, which namely the SIP multi-domain systems, each region has its own independent domain name and SIP server to implement the management of inner domain user [2].

Because of the application of mobile communication network, it can be designed in the application layer to support the user terminal's mobility based on SIP. The support for mobility of SIP implemented by registration mechanism, which register server constantly receive user registration information and update user current address stored in a location server [4]. User identifies current address by register process. With proxy server, redirect server and proper extension, SIP will support users and terminal mobility.

\section{The Overall Structure Design of SIP Multi-domain SmartHome System}

The Network Structure Design of System. As shown in Fig. 1, the SIP Multi-domain SmartHome System consists of two parts: Home External Networks based on Internet and Home Internal Networks based on Home Router. Home external network includes a home SIP server and 
some SIP local terminals (such as PC, embedded fixed terminal and wireless mobile terminal). Home internal network includes SIP Management Network, Application Service and SIP Remote Terminals.

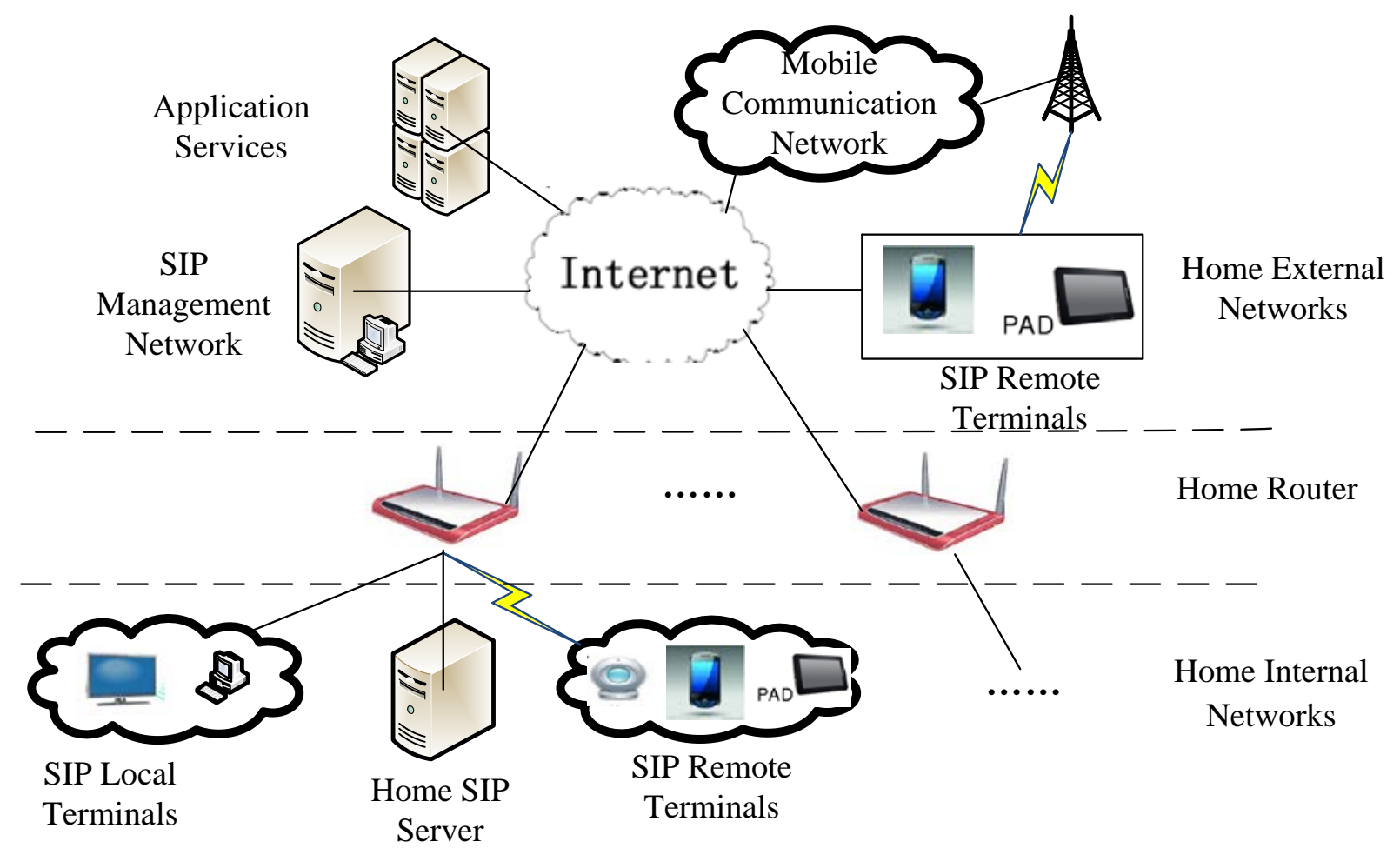

Fig. 1 The Network Architecture of System

Within each home internal network, it is necessary for a SIP server to manage the SIP terminals belonged to the same family group (user group), including the local terminals in the home internal network and the remote terminals in the home external network. After successful registration to the home SIP server, the SIP terminal is possible to communicate each other. With the home external network, there are some SIP register servers and database servers to build a SIP management network, which can manage the home SIP server. The remote terminals can inquiry the information of the home SIP server from the same home group, then realize the functions of Foreign Login and remote-control through the SIP management network. Moreover, Application server to provide value-added services can obtain the user resources by accessing to the database server of the SIP management network.

The Design of SIP Management Network. SIP builds servers to manage network. To support the mobility of the terminal user and the remote access, the structure of servers can adopt distributed server model like domain name server tree. SIP multi-domain systems can be divided into multiple levels, and each level is divided into multiple domains. Server can manage other servers in lower level network domain under its jurisdiction, and provides relocation services to them and SIP terminals.

SIP identifies and locates the SIP user terminal by URL (Uniform Resource Location) [5]. In SIP management network of this system, each server will be assigned a URL according to the domain name it affiliated. The format of URL is: username @ Multi-level domain name it affiliated. In view of the community character of smart home and the family application service, this system can be designed for 3 level domains, as Fig. 2 shows. Top level can be set to $\mathrm{Cn}$ domain according to provincial area. The URL format: Cn @ domain name n, Cn as provincial name, domain name n as DNS domain or public network IP of server. In the actual building process, $\mathrm{Cn}$ can be provincial domain or municipal domain according to the number of users. When the subordinate user scale is too large, we can also add sub-domain to build both provincial and municipal domains at the same time. 
The secondary domain of the middle layer can be set to Dn domain, according to the community, as sub-domains of Cn. The URL format: Dn @ Cn, Dn as community name, Cn as name of provincial domain it affiliated. The third level domain of the bottom level can be set to Hn domain according to family, as sub-domain of Dn. The format of URL: Hn @ Cn.Dn, Hn as family server name, Dn as community server name family affiliated, $\mathrm{Cn}$ as provincial domain name family affiliated. URL format of SIP user will be set as: Un @ Cn.Dn.Hn, Un as username.

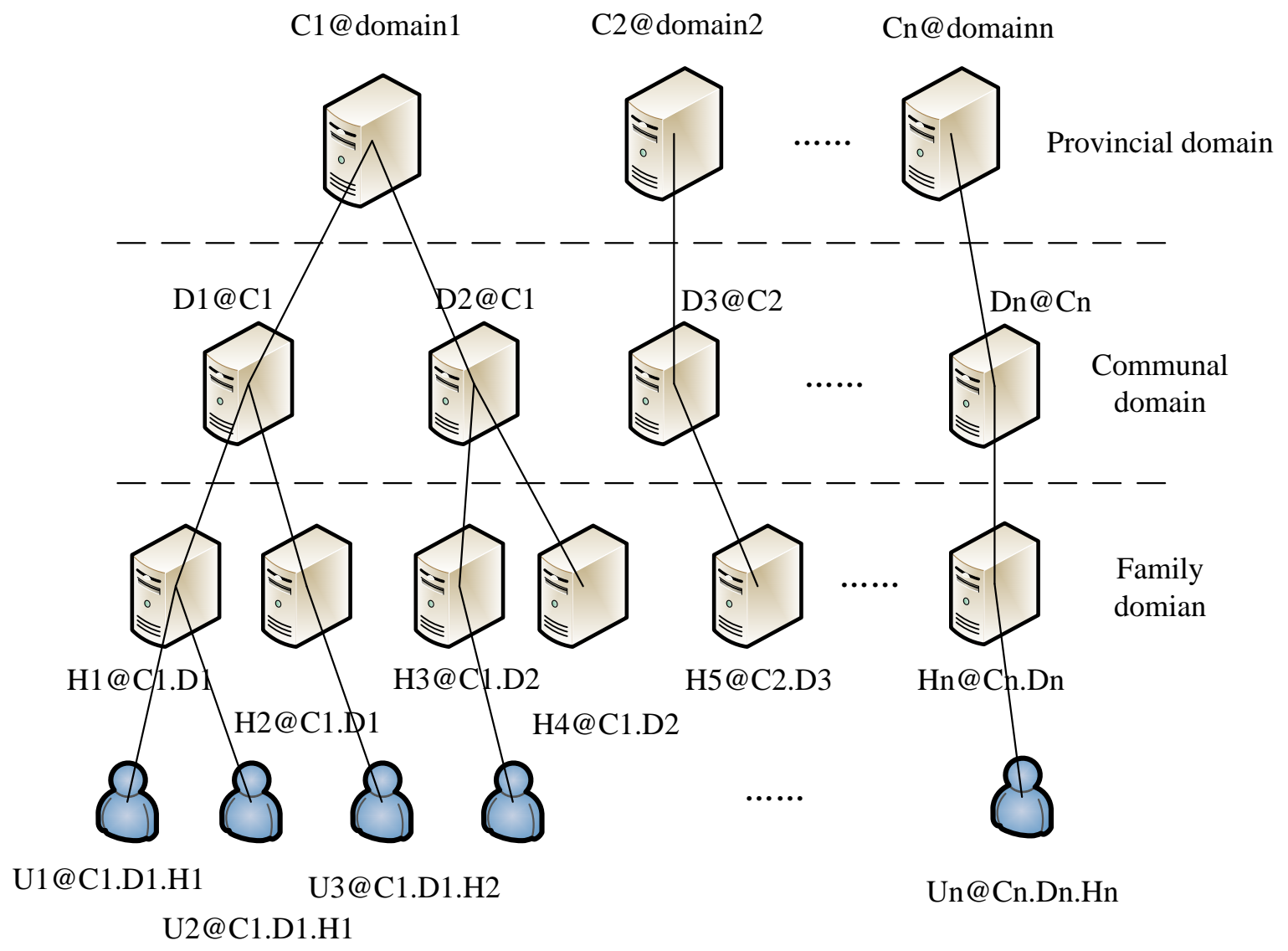

Fig. 2 The hierarchical structure of SIP Management Network

\section{The Designed of SIP Server and Basic Flows}

The Designed of SIP Server. Logically SIP protocol can be divided into the user agent and SIP server, and the SIP server can be divided into register server, agent server, redirect server and location server [5]. These four SIP server is only logical conception, generally, they can be equipped in the same physical entity or selective build-up needed SIP server according to the practical application.

The system in this article is mainly designed for smart home application, the majority communication happens between the SIP terminals within family group. The SIP terminals can realize direct point-to-point communication without transmitted by server if SIP local terminals and SIP remote terminals. This system can achieve the maintenance of session state by SIP terminals without constructing proxy server, which simplify the design and load of SIP server. The software of SIP server adopts the hierarchical design thought, which includes SIP Transaction User Layer, SIP Transaction Layer, SIP Transport Layer and Operating System Support Layer [6]. The software framework is shown in Fig. 3. The top TUL includes register module, authentication module, all state events processing module and redirect module. The register module is consist of the register service side used for receive register order from user or server at a lower level and the register client side used for transmit register order to upper server. The achievement of the three level is based on open source oSIP protocol design, the construction and analysis of SIP message body accomplished, providing SIP TUL responding function interface. 


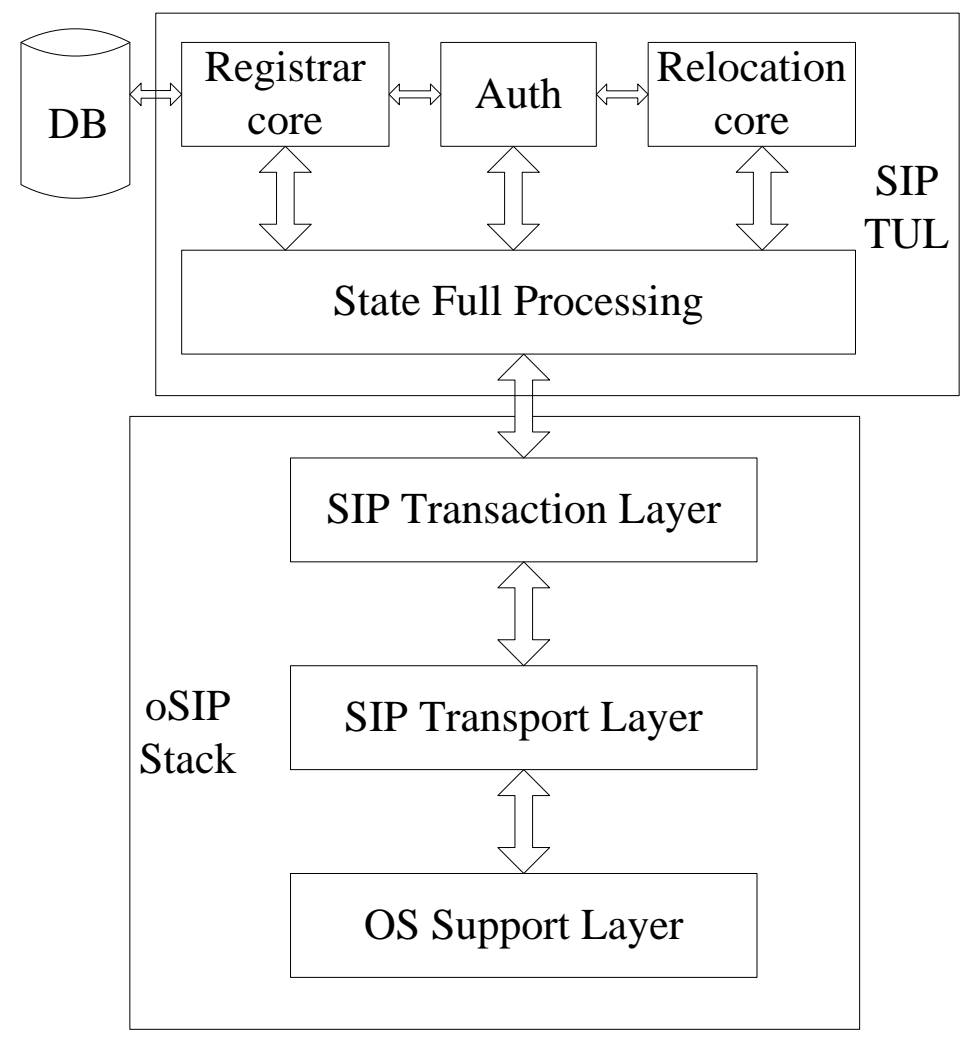

Fig. 3 software architecture of SIP server

The redirect registration and call. As the first step of the system realization, registration flow can establish a binding relationship between the logical address and physical address, then store it to the database [5]. All user accounts can only be registered to the account's family serve in this system. To manage user resource in unit of family, we use a similar tree distributed server model as system networking mode. This paper introduce redirect register mechanism in the standard SIP registration flow, which add redirect answer (302 Moved Temporarily) to REGISTER command and add 302 answer processing to client, as showen in Fig. 4.

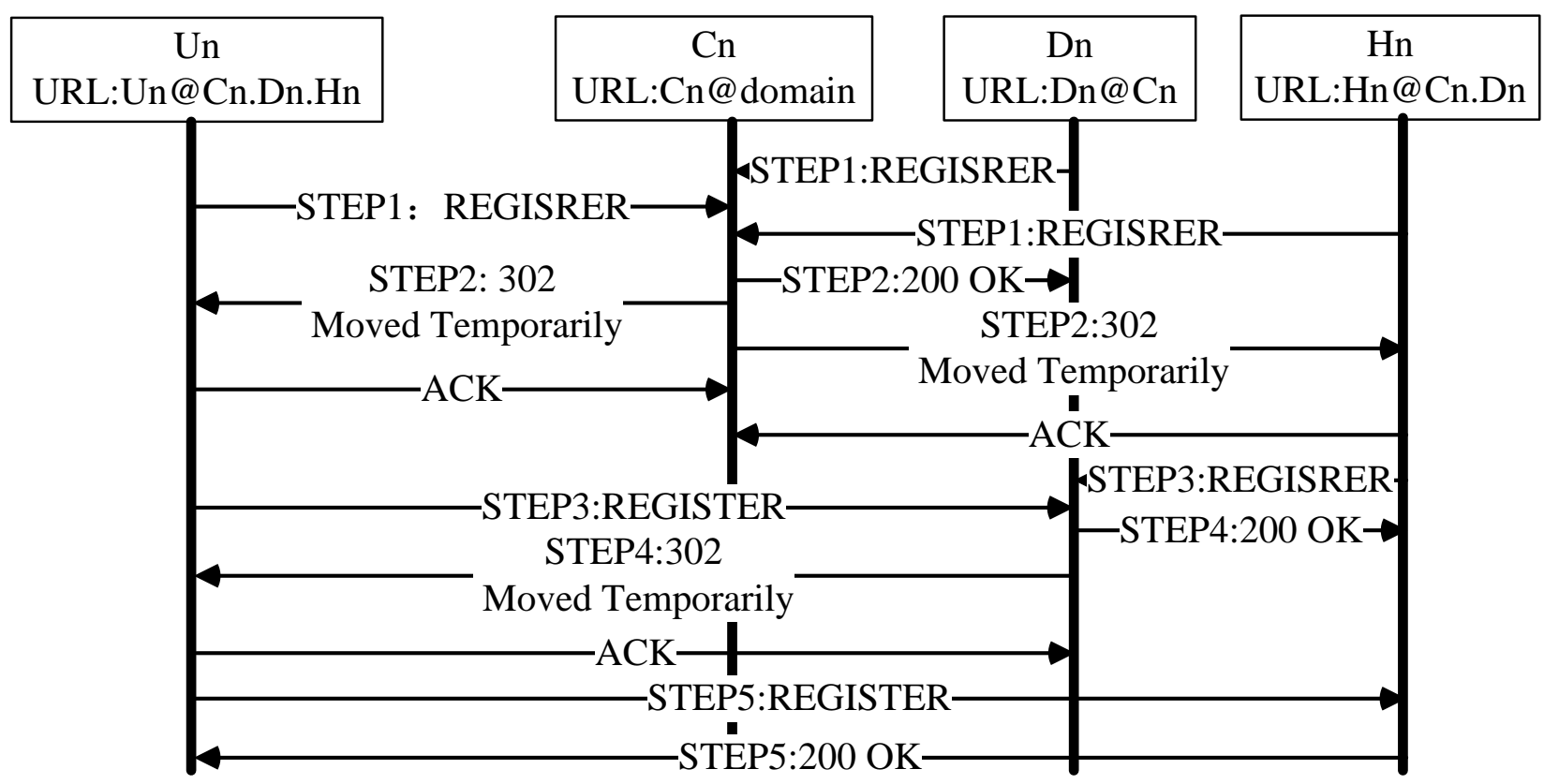

Fig. 4 Redirect registration flow 
STEP1: user terminal(Un), community server(Dn) and family server(Hn) sends REGISTER message to the provincial server(Cn).

STEP2: when the provincial server(Cn) receives the REGISTER message, it gets the URL of sender for the messages, and determines whether the sender is under its jurisdiction. If the sender is a community server under its jurisdiction, the receiver responds with a $200 \mathrm{OK}$ message. If the sender is a family server or user terminal, and its superior server is online, the receiver responds with a 302 Moved Temporarily message. In any other case, the receiver responds with a 404 Not Found message.

STEP3: when a family server(Hn) or user terminal(Un) receives a 302 Moved Temporarily message, it gets the content of CONTACT header field from the message, and then it sends the REGISTER message to the new server (community server) according to the CONTACT header field.

STEP4: when the community server(Dn) receives the REGISTER command, it deals the message as the provincial server.

STEP5: After two redirect registrations, user terminal can successfully log in to the family server, and then send the REGISTER message regularly.

As an important part of system function, The call flow generally contain session establishment, session modification and session termination. There is no proxy server in the system, so the maintaining state of session is realized by client terminals. The specific process shown in Fig. 5.

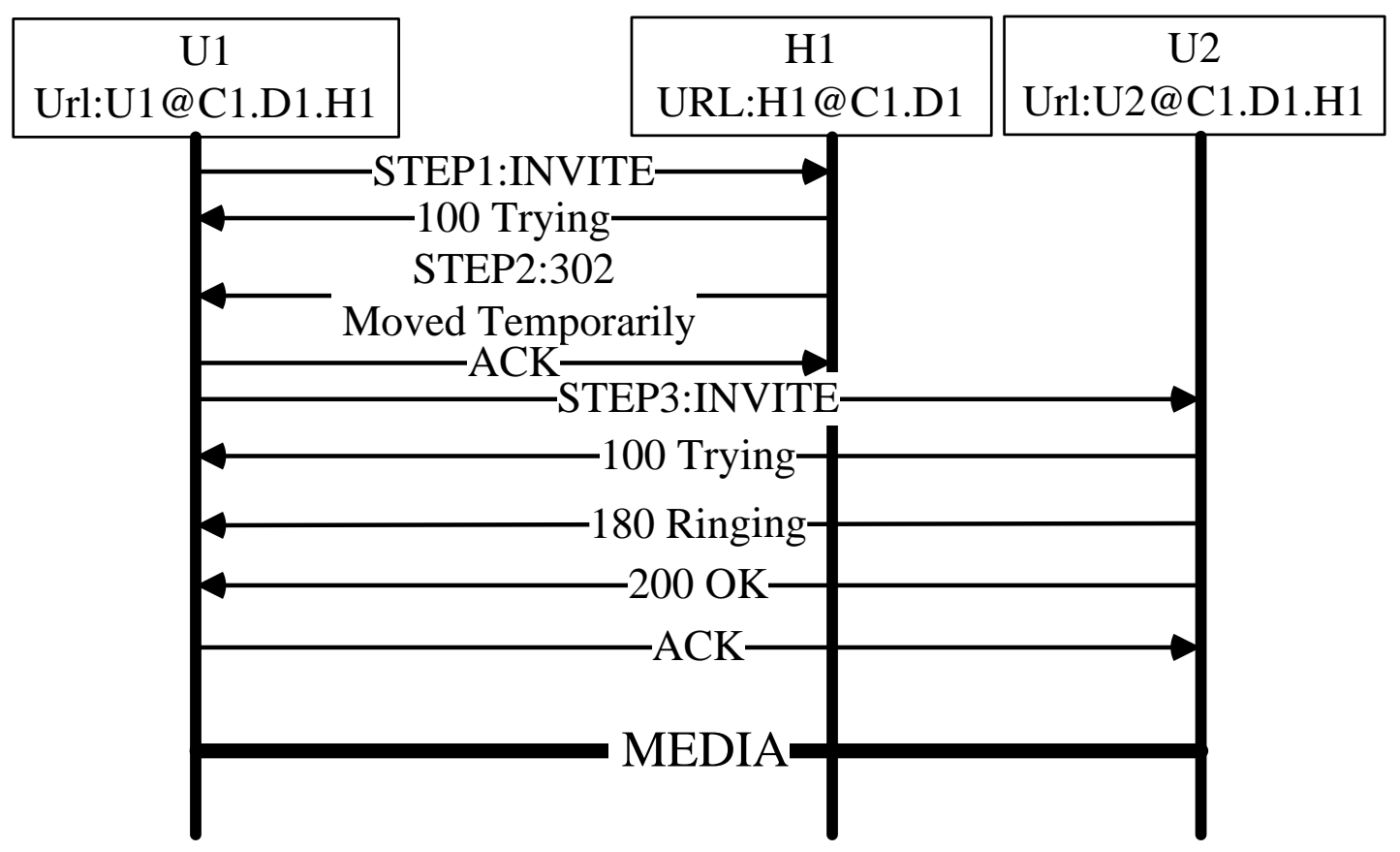

Fig. 5 Redirect call flow

STEP1: user terminal U1 sends INVITE message to family server H1, which contains destination account U2.

STEP2: when family server H1 receives the INVITE message, it gets the location information of the destination account from the location server, and then responds with a 302 Moved Temporarily message.

STEP3: when user terminal U1 receives a 302 Moved Temporarily message, it sends the INVITE message to the user terminal U2 according to the CONTACT header field of the respond message.

STEP4: when user terminal U2 receives the INVITE message, it can make point to point communication with U1, and the call flow is follow the standard procedure of SIP. 


\section{Conclusion}

Aiming at the development and application of smart home, a SIP system with 3 level domains is proposed in this paper. The system makes family as a unit to manage users, builds a stateless SIP server with no proxy and integrated with a registration module which is supposed to be realized in client-side. Subsequently, to reduce the difficulty of development and maintenance costs, the system uses a redirect registration and call, which makes it simpler and more practical. According to the test, it can successfully set up the SIP management network based on configuration documentation once the server is open. And registration for local and remote terminals is OK, therefore, users in the same family network can easily communicate with each other.

As communication among different family networks is rather complicated and should meet the need for greater security, so this article is about neither of that. Additionally, this paper focuses on the study of basic management network, and has not concerned itself more with issues of interrelated application service, all of which will be a further research.

\section{References}

[1] HUANG Shu-rong. Design and Implementation of Information Push Service Platform in Mobile Intelligent District Based on JXTA[D]. MS Thesis of Xiamen University 2014.04.

[2] Shen Qing-guo,LI Xiao-ming,Wang Bin. SIP Multi-domain system and call route query[J]. Journal of Military Communications Technology, Vol.31 No.1 Mar.2010.

[3] Rosenberg J, Schulzrinne H, Camarillo G, et al. SIP: session initiation protocol[R]. RFC 3261, Internet Engineering Task Force, 2002.

[4] ZHOU Da-gang,WAN Lu,LONG Zhao-hua. SIP-based mobility management[J]. Computer Engineering and Design. Vol.26 No.11 Nov.2005.

[5] RFC3263. session initiation protocol(SIP):locating SIP servers[S].

[6] PENG Huan-feng. Research and Design of SIP Registrar. Computer Knowledge and Technology[J]. Vol.6,No.32, November 2010, pp.9024-9026. 